\title{
Retreatment of a Mutilated Tooth with Open Apex by using PRF, MTA and Anatomic Post
}

\author{
Keyur P Chande, Narendra U Manwar, Manoj G Chandak, Jyoti Lokade
}

\begin{abstract}
Apexification aims to induce apical closure of the open root apex with a hard-tissue barrier, against which a root filling can be compacted. Mineral trioxide aggregate (MTA) is a potential apical barrier material with good sealability and a high degree of biocompatibility. The main technical problem associated with placement is to prevent overfill and underfill. This paper demonstrates retreatment of an open apex tooth by placement of an apical barrier using MTA and PRF membrane as an internal matrix followed by application of anatomical post and core. This new technique is particularly advantageous in teeth which have open apex, root canals that are not round, wide canals and thin radicular dentin.
\end{abstract}

Keywords: Retreatment, Apexification, Apical barriers, Internal matrix, Mineral trioxide aggregate, Platelet rich fibrin membrane, Anatomic post.

How to cite this article: Chande KP, Manwar NU, Chandak MG, Lokade J. Retreatment of a Mutilated Tooth with Open Apex by using PRF, MTA and Anatomic Post. Int J Prosthodont Restor Dent 2013;3(3):105-110.

\section{Source of support: Nil}

Conflict of interest: None Declared

\section{INTRODUCTION}

Traumatic injuries in young patients can result in the interruption of the development of the incompletely formed roots . A tooth with a wide canal and blunderbuss open apex may pose several difficulties regarding proper endodontic treatment. Failure resulting from apical leakage is also common due to difficulties in sealing open-apex teeth. Frequently, the extrusion of the root filling materials into the periapical tissues is observed. ${ }^{1}$

Apexification is a method of treatment that induces apical closure of the open root apex with a hard-tissue barrier, against which a root filling can be compacted. Despite the popularity of the apexification procedure calcium hydroxide therapy has some disadvantages that include variability of treatment time, unpredictability of apical closure difficulties with patient follow-up and delayed treatment. Another alternative to the long-term apexification procedure is the use of an artificial apical barrier that allows immediate obturation of the canal. One visit apexification procedure benefits both patient and dentist because of a reduced amount of clinical time required and it forms a good apical seal. This can eliminate the problems associated with extended treatment.

Platelet rich fibrin (PRF) was first described by Choukran et al in France. PRF belongs to a new generation of platelet concentrates, which has been shown to have several advantages like ease of preparation, lack of biochemical handling of blood which makes this preparation strictly autologous, promotion of wound healing, bone growth, bone maturation and hemostasis. ${ }^{2}$

PRF can be obtained in the form of a membrane by squeezing out the fluids in the fibrin clot.

Lemon (1992) introduced the 'internal matrix concept' for treatment of root perforations and also the placement of the restorative material used as artificial barrier was a challenge to prevent it from overfilling and underfilling.

The modified internal matrix concept introduced by Bargohlz uses in contrast to other treatment concepts, collagen as a completely resorbable barrier material and MTA for sealing of the perforation. Repair of perforations requires a matrix to control the repair material. The same concept holds true for placement of the apical barrier in immature tooth.

A number of materials have been proposed for this purpose including tricalcium phosphate, calcium hydroxide, freeze dried bone, freeze dried dentin, collagen calcium phosphate, proplast (a polytetrafluor-ethylene and carbon felt like porous material).

Shabahang et $a l^{3}$ demonstrated that placement of an apical barrier using MTA is a successful alternative to conventional long-term calcium hydroxide therapy. It provides a potential apical barrier material with good sealability and a high degree of biocompatibility.

Prefabricated posts are often used in wide canals due to their ease of placement and a short clinical application time. But in such cases a prefabricated post (metal or fiber) will not simulate the root canal anatomy. An improved option may be the application of chair side fabrication of fiber post; its good adaptation, esthetics and retention are advantageous. As a result of it's precise adaptation to the root canal space, the individualized post is surrounded by thin and uniform layer of resin cement, which creates ideal condition for post retention. ${ }^{4}$

This case report presents the management of an discolored tooth (with an open apex) with a single step apical barrier placement using MTA and autologous PRF membrane as an internal matrix.

\section{CASE REPORT}

A 19-year-old male patient reported to the department of conservative dentistry and endodontics, with the chief 


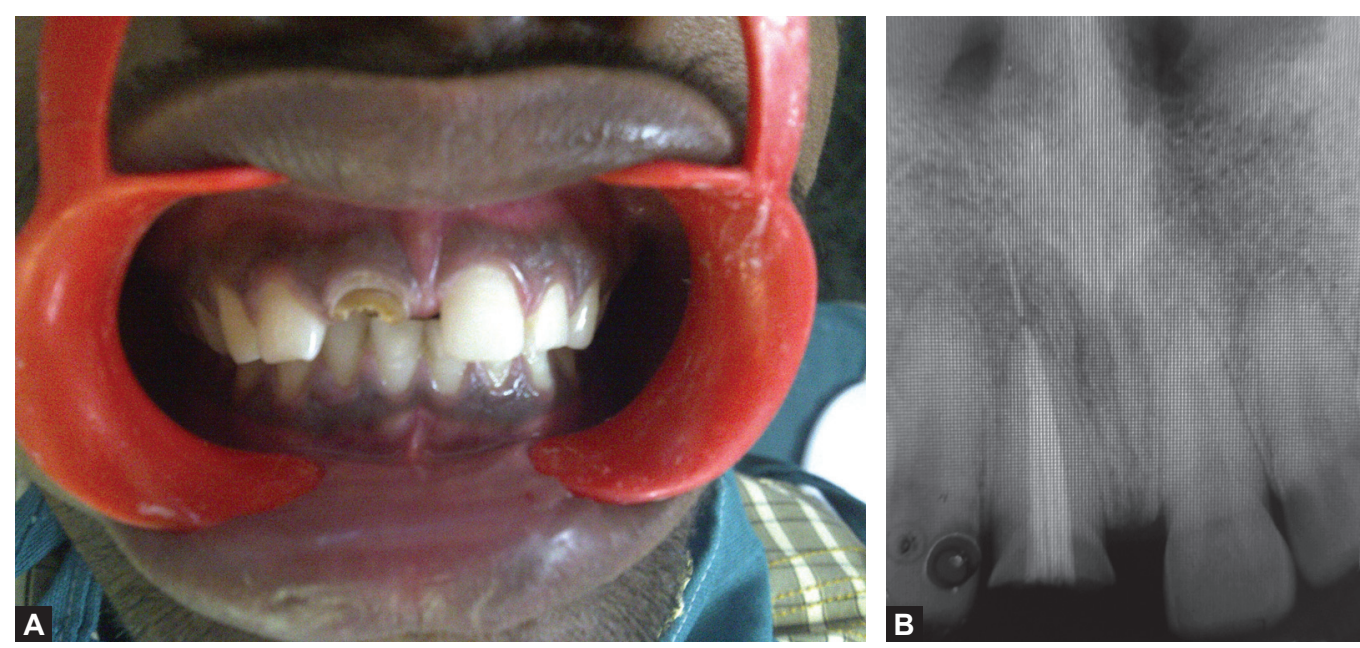

Figs 1A and B: (A) Preoperative clinical view, (B) preoperative radiograph

complaint of discolored and fractured tooth in upper front region of the jaw since 9 years. Dental history revealed that the patient got his tooth fractured due to a trauma 9 years ago for which he had visited a dentist for root canal treatment. Medical history was noncontributory. All vital signs were found to be within normal limits. Oral examination revealed no soft tissue or bony abnormalities.

Extraoral examination was unremarkable. On clinical examination crown fracture was seen with maxillary right central incisor (tooth no. 11) (Fig. 1A) which was not tender to percussion and tested nonvital in response to thermal and electric pulp vitality testing. Radiographic examination revealed a diffuse radiolucency of about $2 \times 3 \mathrm{~mm}$ around the open apex along with some-gutta percha extruded in periapical area (Fig. 1B). It was thus decided to retreat the tooth and to restore it with post and core. Patient was informed about the treatment and its outcome. Informed consent was obtained from the patient.

The patient was concerned about esthetics and considering case it was decided to use artificial root-end barrier procedure with MTA and fiber postanatomic post and core for esthetic rehabilitation of maxillary right central incisor.

After application of rubber dam (Hygienic dental dam, Colténe Whaledent, Germany) and access cavity preparation, working length was determined (Fig. 2). At this stage, size 80 K-file (Dentsply Maillefer, Ballaigues, Switzerland) was loose within the canal and could easily pass beyond the apical limit of the canal. The canal was thoroughly cleaned using 80 K-file (Dentsply Maillefer, Ballaigues, Switzerland) by circumferential filing and $1 \%$ sodium hypochlorite (Dentpro, Chandigarh, India) irrigation and saline $(0.9 \%)$, coupled with ultrasonic agitation (Irrisafe, Satellec, France) to ensure complete removal of the necrotic pulp tissue. To obtain canal disinfection prior to MTA placement, canal was dried with paper points (Dentsply Maillefer, Ballaigues, Switzerland), calcium hydroxide (Ultracal XS, Ultradent, South Jordan and UT) was placed, and the access was closed with a sterile cotton pellet followed by a provisional restorative material IRM (Caulk, Dentsply, Milford, DE) (Fig. 3). The patient was then reappointed 15 days later.

At the next appointment the tooth was re-accessed, and calcium hydroxide was removed. Before obturation, the canal was irrigated with $1 \% \mathrm{NaOCl}, 17 \%$ ethylenediaminetetraacetic acid (Glyde File Prep, Densply, France) to remove the smear layer and the canal was then dried with paper points.

At this appointment, it was decided to use PRF membrane as an internal matrix against which MTA would be placed as an apical barrier.

PRF membrane preparation was performed by using the procedure described by Dohan DM et al 30 minutes before the clinical procedure. $8.5 \mathrm{ml}$ of whole blood was drawn by venipuncture of the antecubital vein. Blood was collected in a $10 \mathrm{ml}$ sterile glass tube without anticoagulant and immediately centrifuged at 3000 revolutions per minute (rpm) for 10 minutes. The resultant product consisted of three layers: topmost layer consisting of acellular platelet poor plasma, PRF clot in the middle and red blood cell's at the bottom. The PRF clot was retrieved and fluids were

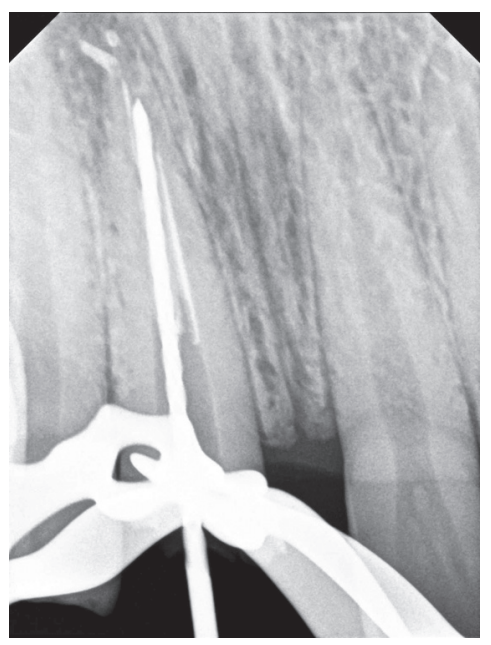

Fig. 2: Diagnostic radiograph 
squeezed out to obtain a PRF membrane (Figs 4 and 5). PRF membrane was gently compacted using hand pluggers to produce a barrier at the level of the apex with the use of operating microscope (Carl Zeiss). White ProRoot MTA (Maillfer, Dentsply, Switzerland) was then mixed with distilled water according to manufacturer's instructions (Fig. 2B) and compacted using schilders pluggers (Dentsply Caulk, Milford, DE) against the PRF membrane. This procedure was repeated a number of times until the thickness of MTA was $4 \mathrm{~mm}$. The plug's position in canal was checked by radiographs. After application of MTA, a wet paper point was left within the canal for 24 hours, and temporary coronal seal was established with IRM (Caulk, Dentsply, Milford, DE). After 3 days, the patient remained asymptomatic and the tooth was isolated and accessed as before.

At the following appointment, restoration of tooth was done with anatomical post and core and metal ceramic crown. The amount of crown structure lost and root canal anatomy did not permit the use of conventional prefabricated fiber post as the post diameter would not have allowed for good adaptation to the post space, and the resulting thick cement

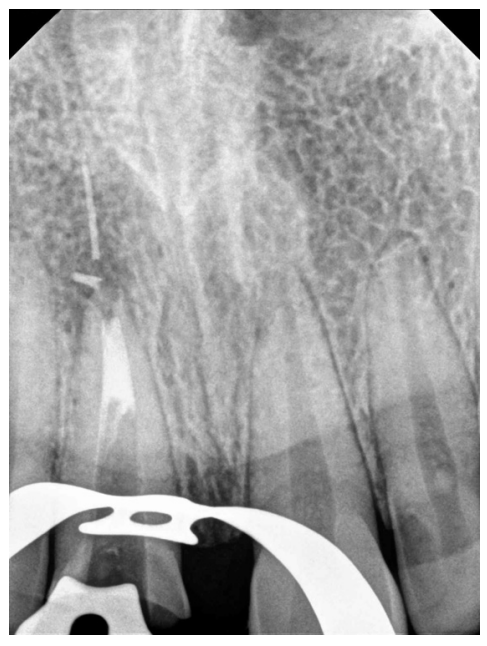

Fig. 3: Calcium hydroxide intracanal dressing

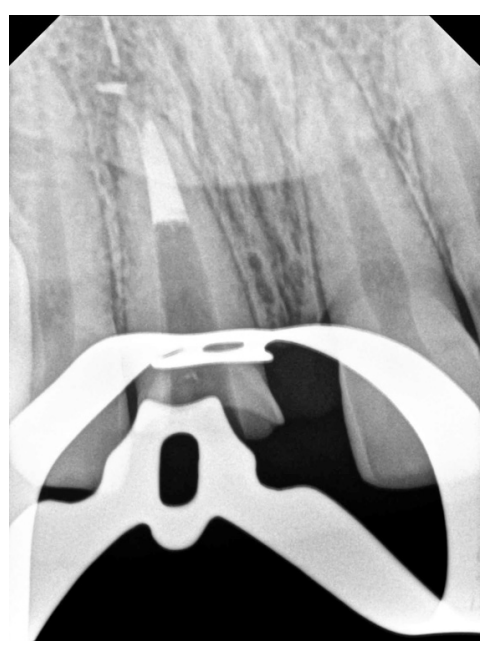

Fig. 4: PRF and MTA plug formed layer would have affected the bond strength. Keeping these factors in mind a decision was made to fabricate an esthetic anatomic post for this tooth. Peeso reamer no. three and four (Dentsply Maillefer, Ballaigues, Switzerland) was used to prepare post space and to remove any undercut that may present on canal walls. The root canal was dried thoroughly and then lubricated with glycerin to act as separator.

A translucent fiber post was selected (Easy Post, Dentsply Maillefer, France) and it was silanized and pretreated with $10 \%$ buffered hydrofluoric acid for 2 minutes followed by the application of silane coupling agent (Ultradent Products Inc, UT, United States), to facilitate the bonding between composite and fiber postmaterial. Resin composite (Filtek $^{\text {TM }}$ P60 3M ESPE, St Paul, MN, USA), was then coated over the post and inserted into the canal adapting it precisely to replicate the canal anatomy.

It was light cured intraorally for 5 seconds and subsequently was withdrawn and light cured extraorally for 20 seconds.

This procedure continued in increments until the post had a snug fit inside the canal. Following this, the core was

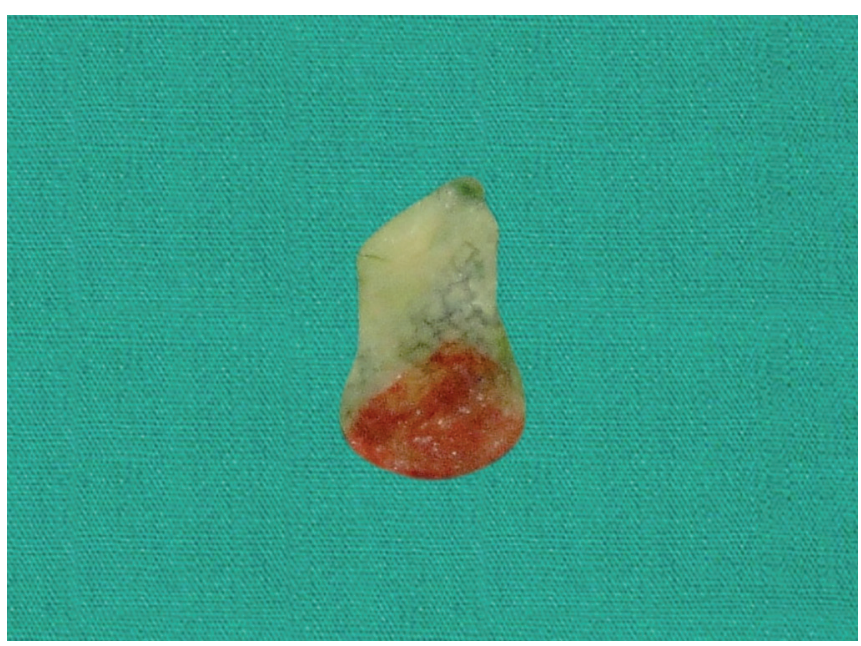

Fig. 5: Platelet rich fibrin membrane (PRF membrane)

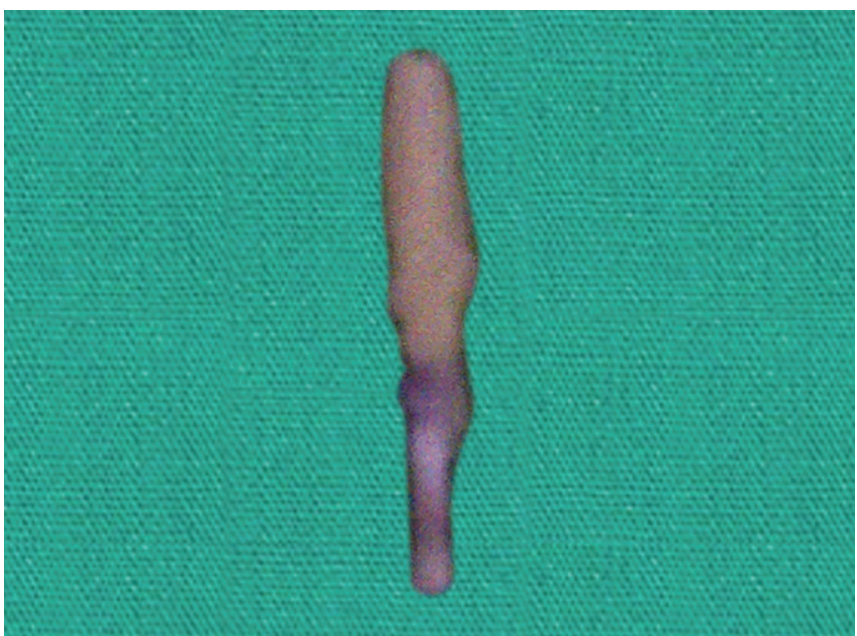

Fig. 6: Anatomic post 


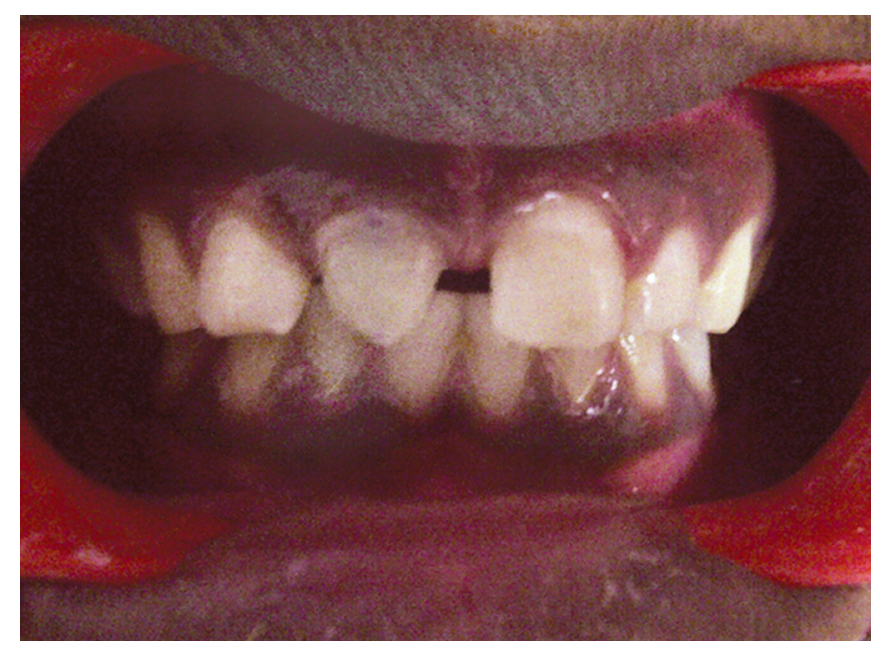

Fig. 7A: A postcementation of post

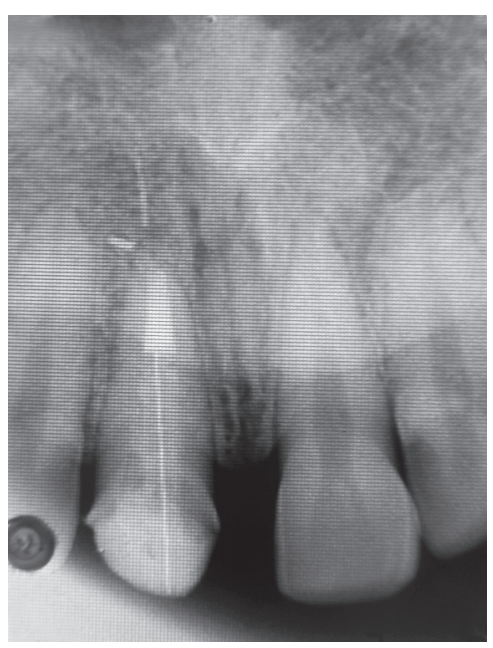

Fig. 7B: Radiograph after postcementation

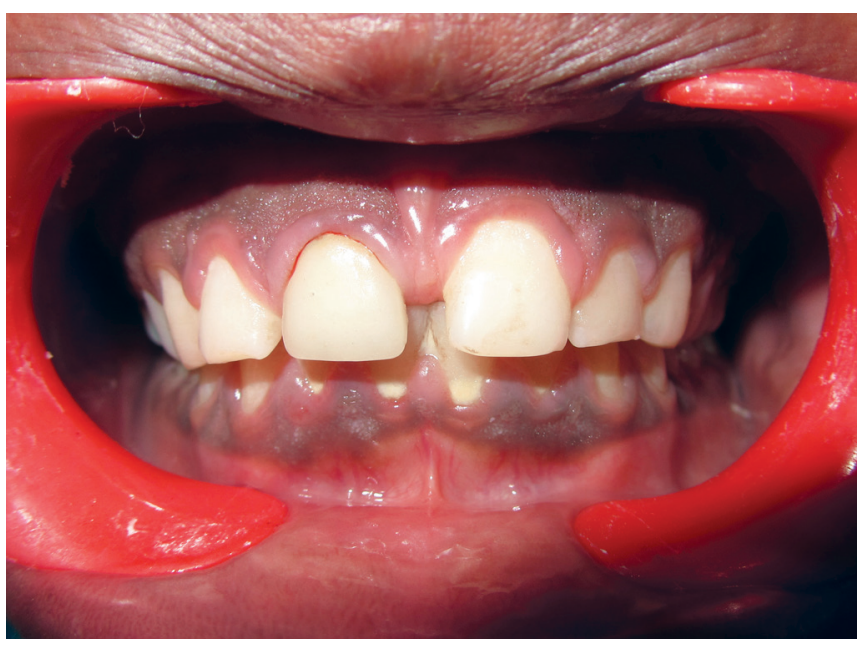

Fig. 8: Clinical view after crown cementation

prepared over the post and light curing was performed in order to completely polymerize the composite.

Post surface was air-abraded with $50 \mu \mathrm{m} \mathrm{Al}_{2} \mathrm{O}_{3}$ abrasive particles for $10 \mathrm{sec}$ prior to cementation to increase surface roughness and surface area for bonding. After sand-blasting, silane was applied to increasing bond strength. A radiograph

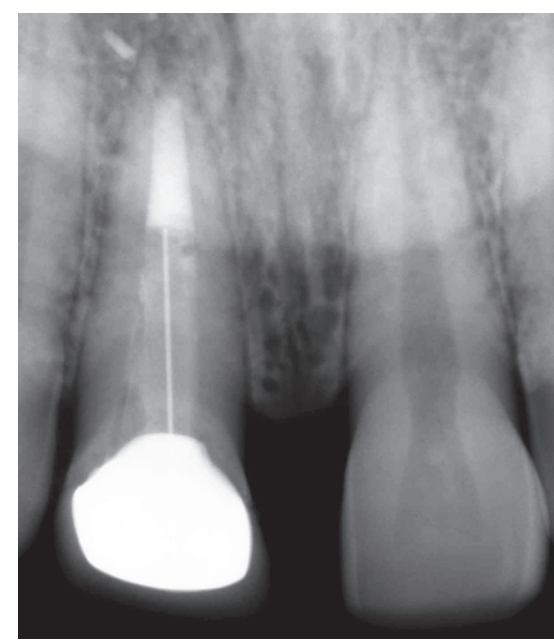

Fig. 9: Six months follow-up radiagraph

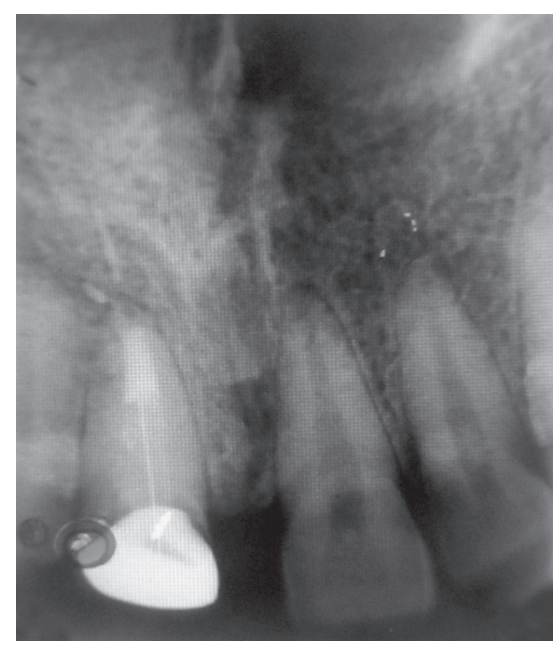

Fig. 10: Nine months follow-up radiograph

was taken to confirm the marginal fit of the anatomical post and core. The root canal walls were etched with $37 \%$ phosphoric acid for 15 seconds and then thoroughly rinsed with water and gently air-dried. The glycerin used as separator was removed at this stage. Bonding agent (Adper Single Bond 2, 3 M ESPE, St. Paul, USA) was applied into the root canal with a microbrush applicator and cured for 20 seconds. Finally, anatomical post and core cemented with dual cure resin cement (Rely-X ARC, 3M ESPE, St Paul, USA) (Fig. 6). A final radiograph was taken to confirm the fit of anatomical post and core into the canal.

Core build-up was done followed by tooth preparation for all-ceramic tooth preparation (Figs 7A and B). Crown cementation was done by dual cure resin cement (Rely-X ARC, 3M ESPE, St Paul, USA) (Fig. 8).

The patient was recalled after 6 and 9 months and no complications were noted. A periapical radiographs demonstrated that anatomical post and core remained well adapted to post space and the complete healing of periapical lesion (Figs 9 and 10). 


\section{DISCUSSION}

The major problem in cases of a wide open apex is the need to limit the material to the apex, thus avoiding the extrusion of a large amount of material into the periodontal tissue. A large volume of the extruded material may set before it disintegrates and gets resorbed. This might result in the persistence of the inflammatory process, which may complicate or even prevent repair of the tissue. Using a matrix avoids the extrusion of the material into the periodontal tissues, reduces leakage in the sealing material, and allows favorable response of the periodontal tissues. Various materials have been used for the formation of matrix like calcium hydroxide, hydroxyapatite, resorbable collagen and calcium sulphate. For the application of MTA, no pressure-resistant support is necessary.

Calcium hydroxide has been the first choice material for apexification, with repeated changes over the course of 5 to 20 months to induce the formation of a calcific barrier. The unpredictable and often lengthy course of this treatment modality presents challenges, including the vulnerability of the temporary coronal restoration to re-infection. Moreover, the treatment requires a high level of patient compliance. For these reasons, one visit apexification has been suggested.

MTA, a biocompatible material, can be used to create a physical barrier that also helps in formation of bone and periodontium around its interface. With the use of MTA, the potential for fractures of immature teeth with thin roots is reduced, because a bonded core can be placed immediately within the root canal. The success rate of apexification with calcium hydroxide is approximately 79 to $96 \%{ }^{5}$

PRF stimulates osteoblasts, gingival fibroblasts and periodontal ligament cells proliferation as a mitogen. Many growth factors such as platelet derived growth factors and transforming growth factors, are released from PRF.

The properties of this natural fibrin biomaterial thus offer great potential during wound healing. ${ }^{6}$ Moreover, PRF is totally autologous and will definitely be more biocompatible than any other material.

Platelets are known to contain a variety of growth factors, including transforming growth factor $\beta$, vascular endothelial growth factor, and platelet-derived growth factor. These growth factors are released from the platelets when they are activated, secreted, or aggregated by collagen or epinephrine. PRF is an immune platelet concentrate, collecting on a single fibrin membrane all the constituents of a blood sample favorable to healing and immunity.

PRF has been considered as a fibrin biomaterial. Its molecular structure with low thrombin concentration is an optimal matrix for migration of endothelial cells and fibroblasts. It permits a rapid angiogenesis and an easier remodeling of fibrin. It features all the necessary parameters permitting optimal healing.

The present case was suitable for including anatomic post as it had very less coronal structure left. Introducing a conventional fiber post into the canal required either force to round off the canal walls or a thick layer of luting cement to fill up the spaces between the loosely fitting post and the canal walls. This would have subjected the restoration and tooth to adhesive failure and/or debonding of the post. Thus using a post which fit canal shape and a thin uniform layer of cement would increase retention. ${ }^{7}$

Esthetic anatomic posts have advantages over the conventional cast metal posts; lower modulus of elasticity protects them from root fracture by reducing the forces that are transferred from the post to the root. A cast metal post would have a wedging effect resulting in root fracture. The esthetic anatomic post also bonds to the tooth structure forming a monobloc unlike a cast metal post. It can also be made in one visit without any laboratory procedures. The disadvantage of this technique is insufficient clinical data regarding the adhesion and endurance of the restoration.

\section{CONCLUSION}

Anatomic post can be used for reconstruction of an endodontically treated tooth when there is substantial loss of crown structure. Combination of 'PRF as a matrix and MTA as an apical barrier' could be considered as a good option for creating artificial periapical seal. However, controlled clinical trials are necessary to investigate the predictability of the outcome of this technique.

\section{REFERENCES}

1. Friedrnan. Treatment outcome and prognosis of endodontic therapy. In: Pitt Ford TR, Orstavik D, editors. Essential endodontology: prevention and treatment of apical periodontitis. Oxford; Blackwell Science 1998;367-391.

2. Dohan DM, Choukroun J, Diss A, Dohan AJ, Mouhi J, et al. Platelet rich fibrin (PRF): A Sencond generation platelet concentrate. Part I: Technological concepts and evolution. Oral Surg Oral Med Oral pathol Oral Radiol Endod 2006;101:E37-44

3. Shabahang, Torabinejad M, Boyne FIP. A comparative study of root-end induction using osteogenic protein-I, calcium hydroxide, and mineral trioxide aggregate in dogs. J Endod 1999;25:1-5.

4. Grandini S, Sapio S, Simonetti M. Use of anatomic post and core for reconstructing an endodontically treated tooth: a case report. J Adhes Dent 2003;5:243-247.

5. Torabinejad M, Pitt Ford TR, McKendry DJ, Abedi HR, Miller DA, Kariyawasam SP. Histologic assessment of mineral trioxide aggregate as a root-end filling in monkeys. J Endod 1997;23: $225-228$ 
6. Tsai CH, Shen SY, Zhao JH, Chang YC. Platelet-rich fibrin modulates cell proliferation of human periodontally related cells in vitro. J Dent Sci 2009;4:130-135.

7. Grandini S, Sapio S, Simonetti M. Use of anatomic post and core for reconstructing an endodontically treated tooth: a case report. J Adhes Dent 2003;5:243-247.

\section{ABOUT THE AUTHORS}

\section{Keyur P Chande (Corresponding Author)}

Postgraduate Student, Department of Conservative Dentistry and Endodontics, Sharad Pawar Dental College, Datta Meghe Institute of Medical Sciences (DU), Wardha, Maharashtra, India, Phone: 09766017629, e-mail: chandekeyur@gmail.com

\section{Narendra U Manwar}

Professor, Department of Conservative Dentistry and Endodontics Sharad Pawar Dental College, Datta Meghe Institute of Medical Sciences (DU), Wardha, Maharashtra, India

\section{Manoj G Chandak}

Professor, Department of Conservative Dentistry and Endodontics Sharad Pawar Dental College, Datta Meghe Institute of Medical Sciences (DU), Wardha, Maharashtra, India

\section{Jyoti Lokade}

Reader, Department of Conservative Dentistry and Endodontics, Sharad Pawar Dental College, Datta Meghe Institute of Medical Sciences (DU), Wardha, Maharashtra, India 